\title{
VIDEO RECOMMENDER IN OPEN/CLOSED SYSTEMS
}

\author{
Ajay Reddy P K ${ }^{1}$, Chidambaram $M^{2}$, D V Anurag ${ }^{3}$, Karthik $S^{4}$, Ravi Teja $K^{5}$, Sri Harish. $\mathbf{N}^{6}$, T. Senthil \\ Kumar $^{7}$ \\ ${ }^{1}$ Student, Computer Science and Engineering, Amrita School of Engineering, Tamil Nadu, India \\ ${ }^{2}$ Student, Computer Science and Engineering, Amrita School of Engineering, Tamil Nadu, India \\ ${ }^{3}$ Student, Computer Science and Engineering, Amrita School of Engineering, Tamil Nadu, India \\ ${ }^{4} S$ Student, Computer Science and Engineering, Amrita School of Engineering, Tamil Nadu, India \\ ${ }^{5}$ Student, Computer Science and Engineering, Amrita School of Engineering, Tamil Nadu, India \\ ${ }^{6}$ Student, Computer Science and Engineering, Amrita School of Engineering, Tamil Nadu, India \\ ${ }^{7}$ Assistant Professor, Computer Science and Engineering Department, Amrita School of Engineering, Tamil Nadu, India
}

\begin{abstract}
The number of people travelling to different countries through airways is increasing day by day. It is the most convenient and fastest way of travelling. Airlines are providing a variety of services to retain customers. Entertainment on board in a flight has become a popular and necessary trend. Airlines often provide a set of movies or videos to the passengers during their travel. But this set of movies may not suit the passenger's mood and needs. Recommended systems helps in suggesting appropriate videos to a particular passenger based on various factors. This will dramatically increase the quality of the service which in turn will increase the popularity of the airline and eventually its revenue. This project comprises of three stages. Firstly, a video of the passenger is captured. Secondly, the captured video is processed for emotion prediction. Subsequently, the emotion along with the passenger details from the airlines is passed onto a system that recommends videos.
\end{abstract}

Keywords: Cloud Computing, Emotion Analysis, Emotion Recognition, Facial Action Coding System, Facial Expression analysis, K-Nearest Neighbor Algorithm, Recommender Systems, Video Processing, Video Recommendation.

$* * *$

\section{INTRODUCTION}

Nowadays, popular services are the ones that customize their service as per the customer's necessities. Each customer is a unique person with their own likes and dislikes. So the services and the software products which understand this uniqueness and provide service make it more successful in today's competitive world. The first step in providing such service is to know what the particular customer needs at that point of time. The next step is to match or customize the service to the determined interests. This paper deals with a solution to a similar problem. Passengers who fly frequently between different countries are often bored in their travel time. The only means of entertainment is watching a select set of videos or movies provided by the airlines. This is similar to television where the user has to surf through a given set of channels and decide which is appropriate for him. But this is tiring since the viewer has to spend his time and effort to browse through the irrelevant content and reach the right video among the huge set of options. This also is uncertain as the viewer may never find anything that matches his interests. Thus the service provided by the airlines is not up to the mark. The passenger might be happier if he can choose among a set of videos which are relevant and interesting to him. The factors that can influence a person's interests and mood are gender, age, nationality, emotion, etc. The key factor in this is the emotion of a person. The videos would be appropriate if it is based on the person's emotion. This paper deals with methods to detect the emotion of a passenger from their facial features. This emotion can then be used by the recommender system to suggest videos. Recommender system facilitates suggestion of appropriate content to a customer based on the factors like emotion, age, gender and nationality. Such systems dramatically increase the quality of service which in turn will increase the popularity of the airline and thereby boost its revenue. The service provider can also charge for streaming the video thereby generating revenue. Since suitable content is favoured over random content this would increase the customer satisfaction than regular videos. This paper discusses about the software comprising of three modules. Firstly, a video of the passenger is captured. The captured video is processed to predict the emotion. Finally, passenger's current emotion along with his details from the airlines reservation is passed onto a recommender system. The recommendations are based on community preferences. Based on user selection, the system learns about the relevance of suggestions and thereby the system improves over time. The recommender system will be running as a cloud service which makes it available anywhere. The video data set can be stored on the cloud storage as a part of DaaS paradigm. Hence, by 
using cloud computing concepts, the system can be made more robust and fail-safe. By using cloud computing models, the system reduces the economic cost involved and also provides reliability.

\section{RELATED WORK}

\subsection{Emotion Analysis}

- Md. Iqbal Et.al [2] work is based on Sobel edge detection. The paper also analyses the accuracy of Fuzzy Logic Edge Detection details of which are discussed in following sections. The paper primarily uses two layered backpropogation neural network. The drawback of this system is that the emotion can be recognised only for the faces that have been mentioned in the research.

- Jeremy N. Bailenson et al. [1] work is based on ChiSquare analysis to find features using distances of major facial features. This system has been implemented only for two emotions, namely happy and sad. Hence this work is not extensible to more expression levels.

- Spiros V. Ioannou et al. [5] work using a hybrid soft computing approach consisting of Nuero-Fuzzy model provides a Detailed Study of how to extract emotional features. The study also provides mathematical models for Nuero-Fuzzy learning.

\subsection{Cloud Computing}

- ShivangiGoyal's [9] work on the cloud services that are currently available in the industry illustrates the advantages and limitations of these services.

- S Rajesh's [10] work describes the usage of cloud products and services. The research shows that the advantages of using cloud outweighs the current limitations.

\subsection{Recommender Systems}

- Tong Zhang's [11] work on Linear Classifiers for Recommender systems show the use of collaborative filtering which is later used in this research.

- Chun Che Fung's [12] research involved a system for suggesting courses to students to take up based on their performances. This work shows the impact of data mining concepts in Recommender Systems.

\section{PROPOSED MODEL}

\subsection{Architecture}

The System is divided into three modules namely, Video Capturing, Emotion prediction, Video recommendations. The video capturing and Emotion prediction is done in a local machine in the flight. After the emotion is predicted, the information is forwarded to the recommender running on the cloud along with passenger information from the Airline Reservations. The recommender queries the video repository. The recommender sends a video list from which the user can select an appropriate video for viewing. The selection by the user is further used to improve the recommender's suggestion over time. The video data set is stored on the cloud using the Infrastructure as a Service (IaaS) and the recommender is run on the cloud (Platform as a Service $(\mathrm{PaaS})$ ).

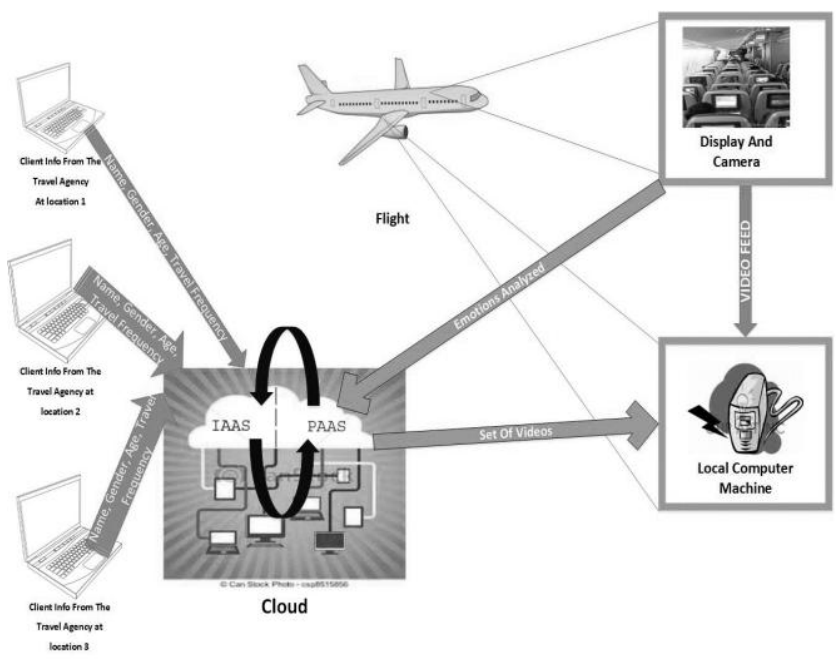

Fig 1 Architecture Diagram

\subsection{Emotion Prediction}

Emotion prediction involves two stages. Facial Feature extraction and the second stage involves these features to predict the emotion.

- For facial feature extraction, the system uses Stasm 3.1[14] for extracting 77 landmark points on a Viola Jones Face [15].

- Based on these feature and Paul Ekman's Facial Action Coding System (FACS) [13], the system can predict whether a person is happy or sad. The system also finds the extent to which a person is happy or sad. This is termed as Emotion Rating which is used in the next section. The Action Units and their mappings are as follows:

Table 1 Emotion to Action Units Mapping

\begin{tabular}{|l|l|}
\hline Emotion & Action Units \\
\hline Happiness & $6+12$ \\
\hline Sadness & $1+4+15$ \\
\hline Surprise & $1+2+5 \mathrm{~B}+26$ \\
\hline Fear & $1+2+4+5+20+26$ \\
\hline Anger & $4+5+7+23$ \\
\hline Disgust & $9+15+16$ \\
\hline Contempt & R12A+R14A \\
\hline
\end{tabular}


Table 2 Action Units for happy and sad emotions

\begin{tabular}{|l|l|l|}
\hline AU & Description & Example Image \\
\hline 1 & Inner Brow Raiser & (O) \\
\hline 4 & Brow Lowerer & \\
\hline 6 & Cheek Raiser & \\
\hline 12 & Lip Corner Puller & \\
\hline & & \\
\hline
\end{tabular}

\subsection{Recommending Videos}

The recommender is constructed on k-Nearest Neighbour (k$\mathrm{NN}$ ) data mining concept. In the constructed recommender the videos are represented as point on a multi-dimensional plot where the plot consists of related parameters. The parameters chosen to construct the recommender are factors that have an influence the choice of videos by an individual for example: emotion, age, gender, language and viewership ratings. These parameters are used to call a WCF service and this service looks up for the points that are plotted near to the point that is referred by the parameters of the user and 9 nearest neighbours to such a point. It is also observed that all factors don't influence the videos to the same degree so the $\mathrm{k}$ nearest neighbour is modified by adding weights so that the most influencing factors contribute to higher distance between the plots in the multi-dimensional plot. These weights increase the efficiency of recommendations since it assigns priorities to the factors. The modified k-NN formula looks as follows:

\section{Distance $=$}

SquareRoot $(50 *$ (PassengerEmotion - VideoEmotion) $*$
$10)+30 *$ (PassengerEmotionRating
VideoEmotionRating)
$+20 *($ Passengergender - VideoGenderPreference $))$
$+10 *($ PassengerAge - Age $) / 12$
$+5 *($ VideoViewership $) / 10^{(\text {maxviewership.Length - 1) })}$

Eq. 1 Modified k-NN Euclidean formula
The weights for each attribute has been decided upon after conducting a video viewership survey based on factors like emotion specific genre, Age group, locale. The survey was conducted among different age groups varying from 10-50 years. The survey was also conducted among some international groups.

\subsection{Learning from the Selection}

The major feature of a recommender is to improve the suggestions over time. When a video is selected by a user it is safe to assume that the suggestion was appropriate and thereby parameters as suitable and closest to the video selected amongst the set of videos. Therefore the video's parameters are modified by averaging the parameters with the history of all the selections so far made. This will keep the influence of the user selection on the video and the suggestions are improved over time. When a new video is inserted the video has no history and no parameters assigned to it so it becomes difficult to profile the video and suggest as it cannot be decided to be relevant to any parameters, therefore they are stored separately. To profile a new incoming video the first suggestion is always said to be a random video from the database so when the user clicks it means he is interested in the video, and the choice can be used to profile the video.

\section{FINDINGS}

The system is divided into two modules namely, Emotion prediction and Recommender System. The emotion prediction involves two stages. Firstly, a video of the passenger is to be captured during the time of take-off of the flight. These videos of each passenger are to be analysed to predict an appropriate emotion. The Second stage involves prediction of the emotion.

- To predict emotion, a face landmark API known as Stasm (Stacked ASM) is used. It is a modification to Active Shape Model where multiple iterations are stacked over the initial Shape model to plot facial feature points. Using these points, based on Paul Ekman's Facial Action Coding System (FACS), emotions are classified into either happy or sad.

- Using Fuzzy Logic, emotions can be predicted as well. But in accordance to Table 1B. Md. Iqbal Quraishi's [2]. research on Human Emotion using Soft computing models, it is found that Fuzzy Logic edge detection provides overlapped minor eye-axis for different emotions. Thereby, hindering the emotion recognition process.

- Chi-square feature selection evaluates the contribution of each feature by computing the value of the Chisquared statistic with respect to emotion ratings. Chisquare method is a Univariate method (It considers one feature at a time). Table 5. Jeremy N. Bailenson Et.al [1]. shows the linear Classification results for different data sets. The work shows the complexity for finding two kinds of emotions, amusement and sadness. 
- It is also found that after using suitable emotion recognition methodology, Genetic algorithm models improves the accuracy of the result comprehensively. Hence, Genetic algorithms can be applied to achieve an optimization.

Recommender Systems have become an integral consequence of increased Business Intelligence in IT industry. There are several algorithms such as k-NN, SVM (Support Vector Machines), ID3 etc. which act as the basis of Recommender Systems. Choosing the appropriate algorithm decides the accuracy and reliability of a Recommendation.

\section{DISCUSSIONS}

As seen in the previous section, there are many ways to conduct emotion analysis for a suitable emotion prediction. To choose a suitable method, we need to compare and contrast each of them based on their advantages and limitations. As mentioned earlier, the Fuzzy logic edge detection approach provides overlapping emotion values. Hence, minor errors in the observed values can lead to wrong emotion prediction. Whenever any Statistical test is used multiple times, then the probability of getting at least one error increases. As a statistical test, Chi square is known to behave erratically for very small expected counts. So to achieve proper results we have to process more than 15,000 instances [1]. The work involves finding codes for expressional changes for a particular emotion. Paul Ekman's FACS has been considered a benchmark for micro-expression analysis. Thus, by using this coding system, detection of happy and sad emotion becomes reliable. Hence, in this paper FACS is considered to be the benchmark for emotion prediction. Recommender Systems uses the modified k-NN clustering technique to find suitable video suggestion. K-NN is a highly extensible model. Thus, if there are any more influential parameters, they can be easily accommodated in the modified k-NN model.

\section{FUTURE ENHANCEMENTS}

The System recommends videos based on two emotions, i.e. happy and sad. Modifications can be made to the system so as to accommodate recommendations based on other emotions such as surprise, anger, disgust, fear, contempt based on Paul Ekman's Facial Action Coding System (FACS). Such a system should be much more accurate in providing recommendations as there are more factors influencing the passenger's emotion.

Videos could be ported to cloud and hence a more personalized video database could be made and would give better content to the viewers. A Recommender running on cloud would provide scalability and reliability as well. In Flight Video Recommender need not be restricted to an airline flight alone. Any public area where there is a specialized display for each person can be used as an application for the recommender system.

\section{CONCLUSIONS}

The implemented system has huge potential in any public place. Computer-Human interactions have become extremely popular. This system is another dimension of how such a system can be used to increase revenue or popularity. The system also collaborates three distinct fields of Computer Sciences namely, Computer Vision, Recommender Systems and Cloud Computing. Hence, it can be concluded that such an integrated system has huge potential in the commercial market.

\section{REFERENCES}

[1] Jeremy N. Bailenson, Emmanuel D. Pontikakis, Iris B. Mauss, James J. Gross, Maria E. Jabon, Cendri A.C. Hutcherson, Clifford Nass, Oliver John. "Real-time classification of evoked emotions using facial feature tracking and physiological responses". In International Jounal of Human-Computer Studies. Vol 66. Int. J. Human-Computer Studies, 2008, 303-317.

[2] Md. Iqbal Quraishi, J Pal Choudhury, Mallika De, PurbajaChakraborty. "A framework for the Recognition of Human Emotion using Soft Computing models". In International Journal of Computer Applications (0975-8887). Vol 40-No.17, February 2012, 50-55.

[3] K B Chandini and Moiz A Hussian. "Emotion Recognition using multilayer perceptron and generalized feed forward neural network". In Journal of Scientific \& Industrial Research. Vol 68, May 2009, 367-371.

[4] DamirFilko and GoranMartinovi. "Emotion Recognition System by a Neural Network Based Facial Expression Analysis. In Automatica". ISSN Print 00051144 vol 54, 2013, 263-272.

[5] SpirosIoannou, LoicKessous, George Caridakis, Kostas Karpouzis, VeredAharonson, StefanosKollias. "Adaptive On-Line Neural Network Retraining for Real Life Multimodal Emotion Recognition".

[6] JasminaNovakovic, MilomirMinic, AlempijeVeljovic. "Classification Accuracy of Neural Networks with PCA in Emotion Recognition". In Theory and Applications of Mathematics \& Computer Science. ISSN Print (2067-2764), 2011, 11-16.

[7] Rana El Kaliouby, Peter Robinson. "Generalization of a Vision-Based Computational Model Of Mindreading". Computer Laboratory, University of Cambridge.

[8] Barry Kort, Rob Reilly, Rosalind W. Picard. “An Affective Model of Interplay between Emotions and Learning: Reengineering Educational PedagogyBuilding a Learning Companion". Massachusetts Institute of Technology.

[9] ShivangiGoyal. "A COMPARATIVE STUDY OF CLOUD COMPUTING SERVICE PROVIDERS”. In International Journal of Advanced Research in 
Computer Science and Software Engineering. ISSN Print: 2277 128X, vol 2, Issue 2, February 2012.

[10] S.Rajesh, S.Swapna, P.Shylender Reddy. "Data as a Service (Daas) in Cloud Computing [Data-As-AService in the Age of Data]". In Global Journal of Computer Science and Technology Cloud \& Distributed. Global Journals Inc. (USA), ISSN Print, Volume 12 Issue 11 Version 1.0,2012.

[11] Tong Zhang, Vijay S. Iyengar.” Recommender Systems Using Linear Classifiers". In Journal of Machine Learning Research. Vol 2, 2002, 313-334.

[12] KANOKWAN KONGSAKUN, CHUN CHE FUNG. "Neural Network Modelling for an Intelligent Recommendation System Supporting SRM for Universities in Thailand". In WSEAS TRANSACTIONS on COMPUTERS. Issue 2, Volume 11, February 2012, 34-44.

[13] P. Ekman and W. V. Friesen. "The Facial Action Coding System". In Consulting Psychologists Press, Inc. 1978.

[14] S. Milborrow and F. Nicolls. "Active Shape Models with SIFT Descriptors and MARS". In VISAPP. 2014

[15] P. Viola and M. Jones. Robust real-time object detection. Jul 2001.

[16] JunghyunAhn, StephaneGobron, Quentin Silvestre, and Daniel Thalmann. "Asymmetrical Facial Expressions based on an Advanced Interpretation of Twodimensional Russell's Emotional Model". In Proceedings of ENGAGE 2010. 2010.

[17] Rong Pan, Peter Dolog, GuandongXu. "KNN-Based Clustering for Improving Social Recommender Systems”. In Springer Berlin Heidelberg. 2013.

[18] Devi Arumugam, Dr. S. Purushothaman. "Emotion Classification Using Facial Expression". In International Journal of Advanced Computer Science and Applications, Vol. 2, No. 7, 2011.

[19] Aleix Martinez, Shichuan Du. "A Model of the Perception of Facial Expressions of Emotion by Humans: Research Overview and Perspectives". Journal of Machine Learning Research 13 (2012) 15891608.

[20] B. Sarwar, G. Karypis, J. Konstan and John Riedl,'Item-based Collaborative Filtering Recommendation Algorithms". Proceedings of 10th international conference on World Wide Web 2001: 285-295.

[21] Y. Zhou, D. Wilkinson, R. Schreiber, R. Pan.LargeScale Parallel Collaborative Filtering for the Netfliix Prize", AAIM 2008: 337-348.

[22] R. Bell, Y. Koren, and C. Volinsky. Modeling relationships at multiple scales to improve accuracy of large recommender systems. In Proc. of KDD '07, pages 95-104, San Jose, California, USA, 2007.

[23] J. Canny. "Collaborative filtering with privacy via factor analysis". In Proc. of SIGIR '02, pages 238-245, Tampere, Finland, 2002.
[24] Gary G. Yen, NethrieNithianandan. "Facial Feature Extraction Using Genetic Algorithm". In IEEE. 2002.

[25] M. Karthigayan, M. Rizon, R. Nagarajan and SazaliYaacob. "Genetic Algorithm and Neural Network for Face Emotion Recognition". In www.intechopen.com.

[26] A. Habibizadnavin, Mir Kamal Mirnia. "A New Algorithm to Classify Face Emotions through Eye and Lip Features by Using Particle Swarm Optimization". In 4th International Conference on Computer Modeling and Simulation (ICCMS 2012). 2012.

[27] Liyanage C De Silva and Suen Chun Hui. "Real-time Facial Feature Extraction and Emotion Recognition". ICICS-PCM 2003 Singapore. 15-18 December 2003.

[28] Chia-Te Liao, Yu-Kuen $\mathrm{Wu}$, and Shang-Hong Lai. "Locating Facial Feature Points Using Support Vector Machines". Department of Computer Science, National Tsing-Hua University, Hsinchu, Taiwan 300, R.O.C. 296-299. 